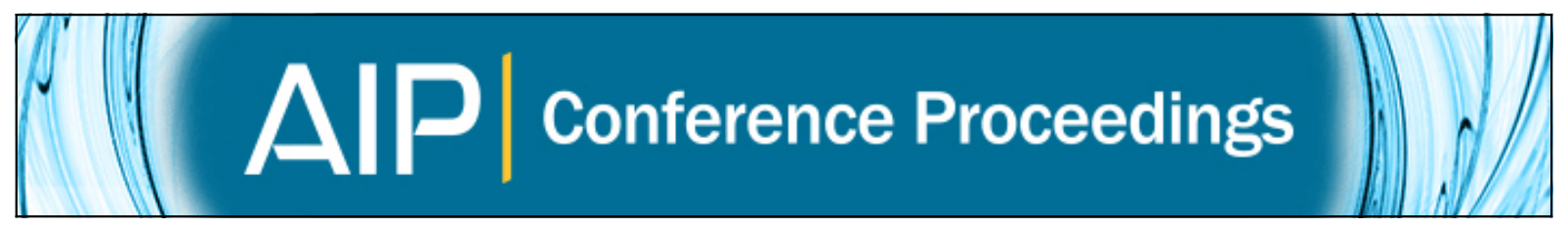

Modeling parameters of the lower and upper bounds and parameters of the objective function for generalized network flow programming problems

L. A. Pilipchuk and A. S. Pilipchuk

Citation: AIP Conference Proceedings 1690, 060007 (2015); doi: 10.1063/1.4936745

View online: http://dx.doi.org/10.1063/1.4936745

View Table of Contents: http://scitation.aip.org/content/aip/proceeding/aipcp/1690?ver=pdfcov

Published by the AIP Publishing

Articles you may be interested in

Upper and lower bounds of the effective trapped particle fraction in general tokamak equilibria

Phys. Plasmas 2, 1666 (1995); 10.1063/1.871315

Upper and Lower Bounds on Static Polarizability

J. Chem. Phys. 55, 5139 (1971); 10.1063/1.1675641

Upper and Lower Bounds to Expectation Values

J. Chem. Phys. 52, 1605 (1970); 10.1063/1.1673176

Upper and Lower Bounds on Knudsen Flow Rates

J. Math. Phys. 8, 514 (1967); 10.1063/1.1705225

Upper and Lower Bounds on Generalized Fourier Coefficients

J. Math. Phys. 5, 1055 (1964); 10.1063/1.1704207 


\title{
MODELING PARAMETERS OF THE LOWER AND UPPER BOUNDS AND PARAMETERS OF THE OBJECTIVE FUNCTION FOR GENERALIZED NETWORK FLOW PROGRAMMING PROBLEMS
}

\author{
L. A. Pilipchuk ${ }^{1, a), b)}$ and A. S. Pilipchuk ${ }^{2, c)}$ \\ ${ }^{1}$ Belarussian State University, 220030 Minsk, 4, Nezavisimosti avenue, Republic of Belarus. \\ ${ }^{2}$ The Natural Resources and Environmental Protestion Ministry of the Republic of Belarus, 220004 Minsk, 10 \\ Kollektornaya Street, Republic of Belarus.

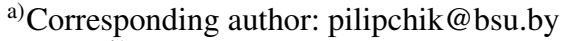 \\ b)URL: http://www.aip.org \\ c)an.pilipchuk@gmail.com
}

\begin{abstract}
In this paper, we propose the mathematical models of the inverse optimization for modeling parameters of the lower and upper bounds and parameters of the objective function for one generalized inhomogeneous linear network flow programming problem under $l_{1}$ norm. We have considered the numerical example of modeling of the parameters of the lower and upper bounds, where new parameters are adjusted as little as possible so, that the infeasible solution becomes the feasible solution for the new values parameters.
\end{abstract}

\section{STATEMENT OF PROBLEM}

Let $G=(I, U)$ be a finite oriented connected multigraph without loops with set of nodes $I$ and set of multiarcs $U$, $|U| \gg|I|$. Let $K(|K|<\infty)$ be a set of different types of flow transported in $G$. We assume that $K=\{1, \cdots,|K|\}$. Let us denote a connected network corresponding to a certain type of flow $k \in K: G^{k}=\left(I^{k}, U^{k}\right), I^{k} \subseteq I, U^{k}=\left\{(i, j)^{k}:(i, j) \in\right.$ $\left.\widehat{U}^{k}\right\}, \widehat{U}^{k} \subseteq U-$ a set of multiarcs of $G$ carrying the flow of type $k \in K$. Also, we define for each multiarc $(i, j) \in U$ the set $K(i, j)=\left\{k \in K:(i, j)^{k} \in U^{k}\right\}$ of types of flow transported through the multiarc $(i, j)$. Consider the following mathematical model:

$$
\begin{gathered}
f(x)=\sum_{(i, j) \in U} \sum_{k \in K(i, j)} c_{i j}^{k} x_{i j}^{k}+\sum_{k \in K} \sum_{i \in I_{k}^{*}} c_{i}^{k} x_{i}^{k} \longrightarrow \text { min, } \\
\sum_{j \in I_{i}^{+}\left(U^{k}\right)} x_{i j}^{k}-\sum_{j \in I_{i}^{-}\left(U^{k}\right)} \mu_{j i}^{k} x_{j i}^{k}=\left\{\begin{array}{ll}
a_{i}^{k}+x_{i}^{k}, & i \in I_{k}^{*}, \\
a_{i}^{k}, & i \in I^{k} \backslash I_{k}^{*},
\end{array} \quad k \in K,\right. \\
\sum_{k \in K} \sum_{(i, j)^{k} \in U^{k}} \lambda_{i j}^{k, p} x_{i j}^{k}+\sum_{k \in K} \sum_{i \in I_{k}^{k}} \lambda_{i}^{k, p} x_{i}^{k}=\beta_{p}, \quad \text { for } \quad p=\overline{1, q}, \\
\sum_{k \in K_{0}(i, j)} x_{i j}^{k} \leq d_{i j}^{0}, x_{i j}^{k} \geq 0, k \in K_{0}(i, j),(i, j) \in U_{0}, \\
0 \leq x_{i j}^{k} \leq d_{i j}^{k}, k \in K_{1}(i, j),(i, j) \in U, \\
x_{i j}^{k} \geq 0, k \in K(i, j) \backslash K_{1}(i, j),(i, j) \in U \backslash U_{0},
\end{gathered}
$$




$$
b_{* i}^{k} \leq x_{i}^{k} \leq b_{i}^{* k}, i \in I_{k}^{*}, k \in K,
$$

where $I_{i}^{+}\left(U^{k}\right)=\left\{j \in I^{k}:(i, j)^{k} \in U^{k}\right\}, I_{i}^{-}\left(U^{k}\right)=\left\{j \in I^{k}:(j, i)^{k} \in U^{k}\right\}, K_{0}(i, j) \subseteq K(i, j), K_{1}(i, j) \subseteq K(i, j)$, $K_{0}(i, j) \cap K_{1}(i, j)=\emptyset, x_{i j}^{k}$ - the flow along the $\operatorname{arc}(i, j)^{k}, a_{i}^{k}, \mu_{i j}^{k}, \lambda_{i j}^{k, p}, \lambda_{i}^{k, p}, \beta_{p}, d_{i j}^{0}, d_{i j}^{k}, b_{* i}^{k}, b_{i}^{* k}$ - known parameters. The nodes $i^{k} \in I_{k}^{*}$ (further $i$ ) are named the nodes with variable intensities $x_{i}^{k}, I_{k}^{*} \subseteq I^{k}, k \in K$.

Flows of different types are interrelated and dependent, since certain types flows using the common power multiarcs. For known values of the parameters in [1] are developed a constructive theory of constructing optimal solutions extremal problem (1) - (7), which is based on the principles of decomposition constraints [2, 3]. Application the principles of decomposition to the construction of solutions of the inhomogeneous problem (1) - (7) is also aimed at the effective solution of the set of homogeneous network tasks using advances in technology of construction their numerical solutions.

\section{INVERSE OPTIMIZATION PROBLEM: MODELING PARAMETERS OF THE LOWER AND UPPER BOUNDS}

Let $x=\left(x_{i j}^{k},(i, j) \in U, k \in K(i, j) ; x_{i}^{k}, i \in I_{k}^{*}, k \in K\right)$ is a infeasible solution of the extremal inhomogeneous problem (1) - (7) (are not met some restrictions of the problem). It is necessary to adjust the values of the lower and upper bounds of the constraints of the problem (1) - (7) so that the infeasible solution $x$ becomes the feasible solution of the problem (1) - (7) for new values of that parameters, where new parameters computed as follows:

$$
\begin{aligned}
& \widetilde{a}_{i}^{k}=a_{i}^{k}+t_{i}^{k}-\psi_{i}^{k}, t_{i}^{k} \geq 0, \psi_{i}^{k} \geq 0, i \in I^{k}, k \in K \\
& \widetilde{\beta}_{p}=\beta_{p}+\varphi_{p}-\delta_{p}, \varphi_{p} \geq 0, \delta_{p} \geq 0, p=\overline{1, q} \\
& \widetilde{d}_{i j}^{0}=d_{i j}^{0}+u_{i j}-h_{i j}, u_{i j} \geq 0, h_{i j} \geq 0,(i, j) \in U_{0} ; \\
& \widetilde{d}_{i j}^{k}=d_{i j}^{k}+m_{i j}^{k}-n_{i j}^{k}, m_{i j}^{k} \geq 0, n_{i j}^{k} \geq 0, k \in K_{1}(i, j),(i, j) \in U ; \\
& \widetilde{b}_{* i}^{k}=b_{* i}^{k}+k_{i}^{k}-s_{i}^{k}, k_{i}^{k} \geq 0, s_{i}^{k} \geq 0, \quad \widetilde{b}_{i}^{* k}=b_{i}^{* k}+u_{i}^{k}-h_{i}^{k}, u_{i}^{k} \geq 0, h_{i}^{k} \geq 0, i \in I_{k}^{*}, k \in K .
\end{aligned}
$$

Values increase and decrease of each parameter for the lower and upper bounds of the constraints to the problem (1) - (7) can not be positive numbers simultaneously.

Applying the principle of inverse optimization $[4,5,6]$ we change the parameters for the upper and lower bounds of the constraints to the problem (1) - (7) in accordance with the following norm $l_{1}$ a sum of vectors:

$$
\begin{aligned}
& \sum_{k \in K} \sum_{i \in I^{k}}\left|t_{i}^{k}-\psi_{i}^{k}\right|+\sum_{p=1}^{q}\left|\varphi_{p}-\delta_{p}\right|+\sum_{(i, j) \in U_{0}}\left|u_{i j}-h_{i j}\right|+ \\
+ & \sum_{(i, j) \in U} \sum_{k \in K_{1}(i, j)}\left|m_{i j}^{k}-n_{i j}^{k}\right|+\sum_{k \in K} \sum_{i \in I_{k}^{k}}\left(\left|k_{i}^{k}-s_{i}^{k}\right|+\left|u_{i}^{k}-h_{i}^{k}\right|\right)= \\
= & \sum_{k \in K} \sum_{i \in I^{k}}\left(t_{i}^{k}+\psi_{i}^{k}\right)+\sum_{p=1}^{q}\left(\varphi_{p}+\delta_{p}\right)+\sum_{(i, j) \in U_{0}}\left(u_{i j}+h_{i j}\right)+ \\
+ & \sum_{(i, j) \in U} \sum_{k \in K_{1}(i, j)}\left(m_{i j}^{k}+n_{i j}^{k}\right)+\sum_{k \in K} \sum_{i \in I_{k}^{k}}\left[\left(k_{i}^{k}+s_{i}^{k}\right)+\left(u_{i}^{k}+h_{i}^{k}\right)\right] .
\end{aligned}
$$

We change the parameters of the upper and lower bounds of the problem (1) - (7) so that the vector $x=$ $\left(x_{i j}^{k},(i, j) \in U, k \in K(i, j) ; x_{i}^{k}, i \in I_{k}^{*}, k \in K\right)$ becomes the feasible solution for the problem (1) - (7) with new modified parameters (8). To change the parameters of the lower and upper bounds for the problem (1) - (7) in accordance with a selected norm, we construct the mathematical model of inverse optimization problem. The inverse optimization problem for the problem (1) - (7) in accordance with the norm $l_{1}$ a sum of vectors has the form:

$$
\sum_{k \in K} \sum_{i \in I^{k}}\left(t_{i}^{k}+\psi_{i}^{k}\right)+\sum_{p=1}^{q}\left(\varphi_{p}+\delta_{p}\right)+\sum_{(i, j) \in U_{0}}\left(u_{i j}+h_{i j}\right)+
$$




$$
\begin{gathered}
+\sum_{(i, j) \in U} \sum_{k \in K_{1}(i, j)}\left(m_{i j}^{k}+n_{i j}^{k}\right)+\sum_{k \in K} \sum_{i \in I_{k}^{*}}\left[\left(k_{i}^{k}+s_{i}^{k}\right)+\left(u_{i}^{k}+h_{i}^{k}\right)\right] \longrightarrow \min , \\
\sum_{j \in I_{i}^{+}\left(U^{k}\right)} x_{i j}^{k}-\sum_{j \in I_{i}^{-}\left(U^{k}\right)} \mu_{j i}^{k} x_{j i}^{k}= \begin{cases}a_{i}^{k}+t_{i}^{k}-\psi_{i}^{k}+x_{i}^{k}, \quad \begin{array}{l}
i \in I_{k}^{*}, \\
a_{i}^{k}+t_{i}^{k}-\psi_{i}^{k}, \quad \\
i \in I^{k} \backslash I_{k}^{*}, \quad t_{i}^{k} \geq 0, \psi_{i}^{k} \geq 0, k \in K ;
\end{array} \\
\sum_{k \in K(i, j)^{k} \in U^{k}} \lambda_{i j}^{k, p} x_{i j}^{k}+\sum_{k \in K} \sum_{i \in I_{k}^{*}} \lambda_{i}^{k, p} x_{i}^{k}=\beta_{p}+\varphi_{p}-\delta_{p}, \varphi_{p} \geq 0, \delta_{p} \geq 0, \quad \text { for } \quad p=\overline{1, q} ; \\
\sum_{k \in K_{0}(i, j)} x_{i j}^{k} \leq d_{i j}^{0}+u_{i j}-h_{i j}, u_{i j} \geq 0, h_{i j} \geq 0, x_{i j}^{k} \geq 0, k \in K_{0}(i, j),(i, j) \in U_{0} ; \\
0 \leq x_{i j}^{k} \leq d_{i j}^{k}+m_{i j}^{k}-n_{i j}^{k}, m_{i j}^{k} \geq 0, n_{i j}^{k} \geq 0, k \in K_{1}(i, j),(i, j) \in U ; \\
x_{i j}^{k} \geq 0, k \in K(i, j) \backslash K_{1}(i, j),(i, j) \in U \backslash U_{0} ;\end{cases} \\
b_{* i}^{k}+k_{i}^{k}-s_{i}^{k} \leq x_{i}^{k} \leq b_{i}^{* k}+u_{i}^{k}-h_{i}^{k}, k_{i}^{k} \geq 0, s_{i}^{k} \geq 0, u_{i}^{k} \geq 0, h_{i}^{k} \geq 0, i \in I_{k}^{*}, k \in K,
\end{gathered}
$$

The unknowns of the inverse optimization problem (9) - (15) are the values of the increase and the decrease of each parameter (8). Decrease and increase of each parameter can not both be positive. The new parameters of the lower and upper bounds adjusted as little as possible so that an infeasible solution $x=\left(x_{i j}^{k},(i, j) \in U, k \in K(i, j) ; x_{i}^{k}, i \in\right.$ $\left.I_{k}^{*}, k \in K\right)$ in the linear optimization problem (1) - (7) becomes the feasible solution for that problem with new parameters (8).

\section{NUMERICAL EXAMPLE OF MODELING PARAMETERS OF THE LOWER AND UPPER BOUNDS}

In Figure 1 a finite connected directed multigraph $G=(I, U)$ is shown, where $|I|=4,|U|=6$ and the nodes with variable intensities forming the set $I_{2}^{*}=\{1\}, I_{4}^{*}=\{4\}$.

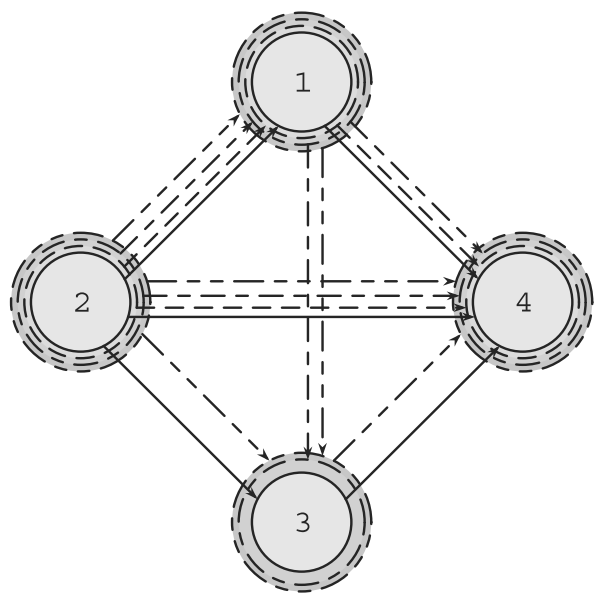

FIGURE 1. Multigraph $G=(I, U),|I|=4,|U|=6, I_{2}^{*}=\{1\}, I_{4}^{*}=\{4\}$.

We present the restrictions and parameters of the generalized network flow programming problem of type (1) (7) for the multigraph (see Figure 1) in the form (16) - (23).

$$
x_{1,4}^{1}-x_{2,1}^{1}=0, \quad x_{2,1}^{1}+x_{2,3}^{1}+x_{2,4}^{1}=15, \quad x_{3,4}^{1}-\frac{9}{10} x_{2,3}^{1}=\frac{16}{5}, \quad-\frac{3}{5} x_{1,4}^{1}-\frac{3}{10} x_{2,4}^{1}-\frac{3}{10} x_{3,4}^{1}=-\frac{63}{10} ;
$$


TABLE 1. Infeasible solution for the known parameters of the restrictions $(16)-(23)$

\begin{tabular}{ccccccccc}
\hline$(i, j), i$ & $(1,3)$ & $(1,4)$ & $(2,1)$ & $(2,3)$ & $(2,4)$ & $(3,4)$ & 1 & 4 \\
\hline$x_{i j}^{1}$ & & 3 & 3 & 5 & 10 & 5 & \\
\hline$x_{i j}^{2}$ & & 1 & 2 & & 2 & & \\
\hline$x_{i j}^{3}$ & 7 & 3 & 9 & & 8 & & \\
\hline$x_{i j}^{4}$ & 6 & & 1 & 0 & 1 & 1 & \\
\hline$x_{1}^{2}$ & & & & & & & 6 \\
\hline$x_{4}^{4}$ & & & & & & & & 1 \\
\hline
\end{tabular}

$$
\begin{aligned}
& x_{1,4}^{2}-\frac{3}{5} x_{2,1}^{2}-x_{1}^{2}=-\frac{1}{5}, \quad x_{2,1}^{2}+x_{2,4}^{2}=4, \quad-\frac{7}{10} x_{1,4}^{2}-\frac{1}{2} x_{2,4}^{2}=-\frac{17}{10} \\
& x_{1,3}^{3}+x_{1,4}^{3}-\frac{1}{10} x_{2,1}^{3}=\frac{47}{5}, \quad x_{2,1}^{3}+x_{2,4}^{3}=14, \quad-\frac{1}{2} x_{1,3}^{3}=-\frac{7}{2}, \quad-\frac{1}{10} x_{1,4}^{3}-x_{2,4}^{3}=-\frac{83}{10} ; \\
& x_{1,3}^{4}-\frac{3}{10} x_{2,1}^{4}=\frac{57}{10}, \quad x_{2,1}^{4}+x_{2,3}^{4}+x_{2,4}^{4}=2, \quad x_{3,4}^{4}-\frac{7}{10} x_{1,3}^{4}-\frac{4}{5} x_{2,3}^{4}=-\frac{16}{5}, \quad-\frac{4}{5} x_{2,4}^{4}-\frac{9}{10} x_{3,4}^{4}-x_{4}^{4}=-\frac{17}{10} \\
& 3 x_{1,3}^{3}+4 x_{1,3}^{4}+x_{1,4}^{1}+3 x_{1,4}^{2}+x_{1,4}^{3}+8 x_{2,1}^{1}+5 x_{2,1}^{2}+3 x_{2,1}^{3}+4 x_{2,1}^{4}+x_{2,3}^{1}+ \\
& +6 x_{2,3}^{4}+9 x_{2,4}^{1}+x_{2,4}^{2}+2 x_{2,4}^{3}+9 x_{2,4}^{4}+2 x_{3,4}^{1}+x_{3,4}^{4}=234, \\
& 8 x_{1,3}^{3}+8 x_{1,3}^{4}+3 x_{1,4}^{1}+3 x_{1,4}^{2}+4 x_{1,4}^{3}+7 x_{2,1}^{1}+5 x_{2,1}^{2}+2 x_{2,1}^{3}+3 x_{2,1}^{4}+7 x_{2,3}^{1}+9 x_{2,3}^{4}+2 x_{2,4}^{1}+5 x_{2,4}^{2}+ \\
& +4 x_{2,4}^{3}+3 x_{2,4}^{4}+9 x_{3,4}^{1}+4 x_{3,4}^{4}=287 \\
& x_{21}^{3}+x_{21}^{4} \leq 8, x_{21}^{3} \geq 0, x_{21}^{4} \geq 0, u_{21} \geq 0, h_{21} \geq 0 \text {, } \\
& x_{2,4}^{1}+x_{2,4}^{2} \leq 15+u_{24}-h_{24}, x_{2,4}^{1} \geq 0, x_{2,4}^{2} \geq 0, u_{24} \geq 0, h_{24} \geq 0 \text {; } \\
& 0 \leq x_{1,3}^{3} \leq 7, \quad 0 \leq x_{1,3}^{4} \leq 10, \quad 0 \leq x_{1,4}^{1} \leq 2, \quad 0 \leq x_{1,4}^{2} \leq 1, \quad 0 \leq x_{1,4}^{3} \leq 3, \\
& 0 \leq x_{2,1}^{1} \leq 2, \quad 0 \leq x_{2,1}^{2} \leq 2, \quad 0 \leq x_{2,1}^{1} \leq 9, \quad 0 \leq x_{2,1}^{4} \leq 1, \quad 0 \leq x_{2,3}^{1} \leq 5, \quad x_{2,3}^{4}=0, \\
& 0 \leq x_{2,4}^{1} \leq 10, \quad 0 \leq x_{2,4}^{2} \leq 2, \quad 0 \leq x_{2,4}^{3} \leq 8, \quad 0 \leq x_{2,4}^{4} \leq 1, \quad 0 \leq x_{3,4}^{1} \leq 5, \quad 0 \leq x_{3,4}^{4} \leq 1, \\
& -5 \leq x_{1}^{2} \leq 5, \quad-2 \leq x_{4}^{4} \leq 2 .
\end{aligned}
$$

In the Table 1 presents an infeasible solution $x=\left(x_{i j}^{k},(i, j) \in U, k \in K(i, j) ; x_{i}^{k}, i \in I_{k}^{*}, k \in K\right)$, where some restrictions (16) - (23) of the considered numerical example are not met for the known parameters. We shall point out 
the parameters for which are not met the restrictions (16) - (23). For the parameters $a_{2}^{1}=15, a_{3}^{1}=\frac{16}{5}, a_{1}^{2}=-\frac{1}{5}$, $a_{1}^{3}=\frac{47}{5}, a_{2}^{3}=14, a_{4}^{3}=-\frac{83}{10}$ and $a_{4}^{4}=-\frac{17}{10}$ are not met the following restrictions:

$$
\begin{gathered}
x_{2,1}^{1}+x_{2,3}^{1}+x_{2,4}^{1}=15, \quad x_{3,4}^{1}-\frac{9}{10} x_{2,3}^{1}=\frac{16}{5}, \quad x_{1,4}^{2}-\frac{3}{5} x_{2,1}^{2}-x_{1}^{2}=-\frac{1}{5}, \quad x_{1,3}^{3}+x_{1,4}^{3}-\frac{1}{10} x_{2,1}^{3}=\frac{47}{5} \\
x_{2,1}^{3}+x_{2,4}^{3}=14, \quad-\frac{1}{10} x_{1,4}^{3}-x_{2,4}^{3}=-\frac{83}{10}, \quad-\frac{4}{5} x_{2,4}^{4}-\frac{9}{10} x_{3,4}^{4}-x_{4}^{4}=-\frac{17}{10} .
\end{gathered}
$$

The restrictions (20) are not met for the parameters $\beta_{1}=234$ and $\beta_{2}=287$. The restriction $x_{2,1}^{3}+x_{2,1}^{4} \leq 8$ is not met for the parameter $d_{2,1}^{0}=8$. The following restrictions are not satisfied for the known parameters $d_{1,4}^{1}=2$ and $d_{2,1}^{1}=2$ : $0 \leq x_{1,4}^{1} \leq 2, \quad 0 \leq x_{2,1}^{1} \leq 2$. The restriction $-5 \leq x_{1}^{2} \leq 5$ is not met for the parameters $b_{* 1}^{2}=-5$ and $b_{1}^{* 2}=5$.

Mathematical model of the inverse optimization problem for the modeling of the lower and upper bounds has the form $(25)-(33)$.

$$
\begin{aligned}
& \sum_{k=1,3,4} \sum_{i=1,2,3,4}\left(t_{i}^{k}+\psi_{i}^{k}\right)+\sum_{i=1,2,4}\left(t_{i}^{2}+\psi_{i}^{2}\right)+\sum_{p=1}^{2}\left(\varphi_{p}+\delta_{p}\right)+ \\
& +\left(u_{2,1}+h_{2,1}\right)+\left(u_{2,4}+h_{2,4}\right)+\sum_{k=3,4}\left(m_{1,3}^{k}+n_{1,3}^{k}\right)+\sum_{k=1,2,3}\left(m_{1,4}^{k}+n_{1,4}^{k}\right)+ \\
& +\sum_{k=1,2,3,4}\left(m_{2,1}^{k}+n_{2,1}^{k}\right)+\sum_{k=1,2,3,4}\left(m_{2,4}^{k}+n_{2,4}^{k}\right)+\sum_{k=1,4}\left(m_{2,3}^{k}+n_{2,3}^{k}\right)+\sum_{k=1,4}\left(m_{3,4}^{k}+n_{3,4}^{k}\right)+ \\
& +\left(k_{1}^{2}+s_{1}^{2}\right)+\left(u_{1}^{2}+h_{1}^{2}\right)+\left(k_{4}^{4}+s_{4}^{4}\right)+\left(u_{4}^{4}+h_{4}^{4}\right) \longrightarrow \min , \\
& x_{1,4}^{1}-x_{2,1}^{1}=t_{1}^{1}-\psi_{1}^{1}, t_{1}^{1} \geq 0, \psi_{1}^{1} \geq 0, \\
& x_{2,1}^{1}+x_{2,3}^{1}+x_{2,4}^{1}=15+t_{2}^{1}-\psi_{2}^{1}, t_{2}^{1} \geq 0, \psi_{2}^{1} \geq 0 \\
& x_{3,4}^{1}-\frac{9}{10} x_{2,3}^{1}=\frac{16}{5}+t_{3}^{1}-\psi_{3}^{1}, t_{3}^{1} \geq 0, \psi_{3}^{1} \geq 0, \\
& -\frac{3}{5} x_{1,4}^{1}-\frac{3}{10} x_{2,4}^{1}-\frac{3}{10} x_{3,4}^{1}=-\frac{63}{10}+t_{4}^{1}-\psi_{4}^{1}, t_{4}^{1} \geq 0, \psi_{4}^{1} \geq 0, \\
& x_{1,4}^{2}-\frac{3}{5} x_{2,1}^{2}-x_{1}^{2}=-\frac{1}{5}+t_{1}^{2}-\psi_{1}^{2}, t_{1}^{2} \geq 0, \psi_{1}^{2} \geq 0, \\
& x_{2,1}^{2}+x_{2,4}^{2}=4+t_{2}^{2}-\psi_{2}^{2}, t_{2}^{2} \geq 0, \psi_{2}^{2} \geq 0, \\
& -\frac{7}{10} x_{1,4}^{2}-\frac{1}{2} x_{2,4}^{2}=-\frac{17}{10}+t_{4}^{2}-\psi_{4}^{2}, t_{4}^{2} \geq 0, \psi_{4}^{2} \geq 0 \text {, }
\end{aligned}
$$




$$
\begin{aligned}
& x_{1,3}^{3}+x_{1,4}^{3}-\frac{1}{10} x_{2,1}^{3}=\frac{47}{5}+t_{1}^{3}-\psi_{1}^{3}, t_{1}^{3} \geq 0, \psi_{1}^{3} \geq 0, \\
& x_{2,1}^{3}+x_{2,4}^{3}=14+t_{2}^{3}-\psi_{2}^{3}, t_{2}^{3} \geq 0, \psi_{2}^{3} \geq 0, \\
& -\frac{1}{2} x_{1,3}^{3}=-\frac{7}{2}+t_{3}^{3}-\psi_{3}^{3}, t_{3}^{3} \geq 0, \psi_{3}^{3} \geq 0, \\
& -\frac{1}{10} x_{1,4}^{3}-x_{2,4}^{3}=-\frac{83}{10}+t_{4}^{3}-\psi_{4}^{3}, t_{4}^{3} \geq 0, \psi_{4}^{3} \geq 0, \\
& x_{1,3}^{4}-\frac{3}{10} x_{2,1}^{4}=\frac{57}{10}+t_{1}^{4}-\psi_{1}^{4}, t_{1}^{4} \geq 0, \psi_{1}^{4} \geq 0, \\
& x_{2,1}^{4}+x_{2,3}^{4}+x_{2,4}^{4}=2+t_{2}^{4}-\psi_{2}^{4}, t_{2}^{4} \geq 0, \psi_{2}^{4} \geq 0, \\
& x_{3,4}^{4}-\frac{7}{10} x_{1,3}^{4}-\frac{4}{5} x_{2,3}^{4}=-\frac{16}{5}+t_{3}^{4}-\psi_{3}^{4}, t_{3}^{4} \geq 0, \psi_{3}^{4} \geq 0, \\
& -\frac{4}{5} x_{2,4}^{4}-\frac{9}{10} x_{3,4}^{4}-x_{4}^{4}=-\frac{17}{10}+t_{4}^{4}-\psi_{4}^{4}, t_{4}^{4} \geq 0, \psi_{4}^{4} \geq 0, \\
& 3 x_{1,3}^{3}+4 x_{1,3}^{4}+x_{1,4}^{1}+3 x_{1,4}^{2}+x_{1,4}^{3}+8 x_{2,1}^{1}+5 x_{2,1}^{2}+3 x_{2,1}^{3}+4 x_{2,1}^{4}+x_{2,3}^{1}+ \\
& +6 x_{2,3}^{4}+9 x_{2,4}^{1}+x_{2,4}^{2}+2 x_{2,4}^{3}+9 x_{2,4}^{4}+2 x_{3,4}^{1}+x_{3,4}^{4}=234+\varphi_{1}-\delta_{1}, \varphi_{1} \geq 0, \delta_{1} \geq 0, \\
& 8 x_{1,3}^{3}+8 x_{1,3}^{4}+3 x_{1,4}^{1}+3 x_{1,4}^{2}+4 x_{1,4}^{3}+7 x_{2,1}^{1}+5 x_{2,1}^{2}+2 x_{2,1}^{3}+3 x_{2,1}^{4}+7 x_{2,3}^{1}+9 x_{2,3}^{4}+2 x_{2,4}^{1}+5 x_{2,4}^{2}+ \\
& +4 x_{2,4}^{3}+3 x_{2,4}^{4}+9 x_{3,4}^{1}+4 x_{3,4}^{4}=287+\varphi_{2}-\delta_{2}, \varphi_{2} \geq 0, \delta_{2} \geq 0 ; \\
& x_{2,1}^{3}+x_{2,1}^{4} \leq 8+u_{2,1}-h_{2,1}, x_{2,1}^{3} \geq 0, x_{2,1}^{4} \geq 0, u_{2,1} \geq 0, h_{2,1} \geq 0, \\
& x_{2,4}^{1}+x_{2,4}^{2} \leq 15+u_{2,4}-h_{2,4}, x_{2,4}^{1} \geq 0, x_{2,4}^{2} \geq 0, u_{2,4} \geq 0, h_{2,4} \geq 0 \text {; } \\
& 0 \leq x_{2,3}^{4} \leq m_{2,3}^{4}-n_{2,3}^{4}, \quad m_{2,3}^{4} \geq 0, \quad n_{2,3}^{4} \geq 0, \quad 0 \leq x_{2,4}^{1} \leq 10+m_{2,4}^{1}-n_{2,4}^{1}, \quad m_{2,4}^{1} \geq 0, n_{2,4}^{1} \geq 0, \\
& 0 \leq x_{2,4}^{2} \leq 2+m_{2,4}^{2}-n_{2,4}^{2}, \quad m_{2,4}^{2} \geq 0, n_{2,4}^{2} \geq 0, \quad 0 \leq x_{2,4}^{3} \leq 8+m_{2,4}^{3}-n_{2,4}^{3}, \quad m_{2,4}^{3} \geq 0, \quad n_{2,4}^{3} \geq 0, \\
& 0 \leq x_{2,4}^{4} \leq 1+m_{2,4}^{4}-n_{2,4}^{4}, \quad m_{2,4}^{4} \geq 0, n_{2,4}^{4} \geq 0, \quad 0 \leq x_{3,4}^{1} \leq 5+m_{3,4}^{1}-n_{3,4}^{1}, \quad m_{3,4}^{1} \geq 0, \quad n_{3,4}^{1} \geq 0 ; \\
& 0 \leq x_{3,4}^{4} \leq 1+m_{3,4}^{4}-n_{3,4}^{4}, \quad m_{3,4}^{4} \geq 0, \quad n_{3,4}^{4} \geq 0 ;
\end{aligned}
$$




$$
\begin{aligned}
& -5+k_{1}^{2}-s_{1}^{2} \leq x_{1}^{2} \leq 5+u_{1}^{2}-h_{1}^{2}, \quad k_{1}^{2} \geq 0, \quad s_{1}^{2} \geq 0, \quad u_{1}^{2} \geq 0, \quad h_{1}^{2} \geq 0, \\
& -2+k_{4}^{4}-s_{4}^{4} \leq x_{4}^{4} \leq 2+u_{4}^{4}-h_{4}^{4}, \quad k_{4}^{4} \geq 0, \quad s_{4}^{4} \geq 0, \quad u_{4}^{4} \geq 0, \quad h_{4}^{4} \geq 0 .
\end{aligned}
$$

The new parameters (8) for the problem (25) - (33) computed as follows:

$$
\begin{gathered}
\widetilde{a}_{i}^{k}=a_{i}^{k}+t_{i}^{k}-\psi_{i}^{k}, t_{i}^{k} \geq 0, \psi_{i}^{k} \geq 0, i \in I^{k}, k \in K=\{1,2,3,4\} ; \quad \widetilde{\alpha}_{p}=\alpha_{p}+\varphi_{p}-\delta_{p}, \varphi_{p} \geq 0, \delta_{p} \geq 0, p=\overline{1,2} ; \\
\widetilde{d}_{i j}^{0}=d_{i j}^{0}+u_{i j}-h_{i j}, u_{i j} \geq 0, h_{i j} \geq 0,(i, j) \in U_{0}=\{(2,1),(2,4)\} ; \\
\widetilde{d}_{i j}^{k}=d_{i j}^{k}+m_{i j}^{k}-n_{i j}^{k}, m_{i j}^{k} \geq 0, n_{i j}^{k} \geq 0,(i, j)^{k} \in T, \\
T\left\{(1,3)^{k}, k=3,4 ;(1,4)^{k}, k=1,2,3 ;(2,1)^{k}, k=1,2,3,4 ;(2,4)^{k}, k=1,2,3,4 ;(2,3)^{k}, k=1,4 ;(3,4)^{k}, k=1,4\right\} ; \\
\widetilde{b}_{* i k}=b_{* i}^{k}+k_{i}^{k}-s_{i}^{k}, k_{i}^{k} \geq 0, s_{i}^{k} \geq 0, \quad \widetilde{b}_{i}^{* k}=b_{i}^{* k}+u_{i}^{k}-h_{i}^{k}, u_{i}^{k} \geq 0, h_{i}^{k} \geq 0, i \in I_{k}^{*}, I_{2}^{*}=\{1\}, \quad I_{4}^{*}=\{4\} .
\end{gathered}
$$

The values increase and decrease of each known parameter of lower and upper bounds are the unknowns of the problem (25) - (33). Decrease and increase of each parameter can not both be positive.

As a result of solving the inverse optimization problem (25) - (33) we obtained the following changes of the parameters :

$$
\begin{gathered}
t_{2}^{1}=3, \quad \psi_{3}^{1}=\frac{27}{10}, \quad \psi_{1}^{2}=6, \quad \psi_{1}^{3}=\frac{3}{10}, \quad t_{2}^{3}=3, \quad t_{4}^{3}=\frac{107}{10}, \quad \psi_{4}^{4}=1, \\
\varphi_{1}=18, \quad \varphi_{2}=42, \quad u_{2,1}=2, \quad m_{1,4}^{1}=1, \quad m_{2,1}^{1}=1, \quad u_{1}^{2}=1 .
\end{gathered}
$$

The parameters of lower and upper bounds adjusted as little as possible so that an infeasible solution $x=$ $\left(x_{i j}^{k},(i, j) \in U, k \in K(i, j) ; x_{i}^{k}, i \in I_{k}^{*}, k \in K\right)$ becomes the feasible solution for the new parameters. For the vector $x$ (see Table 1) fulfilled all restrictions (16) - (23), therefore $x$ is a feasible solution for the new parameters in the considered numerical example.

\section{INVERSE OPTIMIZATION PROBLEM: MODELING PARAMETERS OF THE OBJECTIVE FUNCTION}

For the primal problem (1) - (7) we form the dual problem (34) - (38):

$$
\begin{gathered}
\sum_{k \in K} \sum_{i \in I^{k}} a_{i}^{k} u_{i}^{k}+\sum_{p=1}^{q} \beta_{p} r_{p}-\sum_{(i, j) \in U_{0}} d_{i j}^{0} v_{i j}-\sum_{(i, j) \in U} \sum_{k \in K_{1}(i, j)} d_{i j}^{k} \omega_{i j}^{k}+\sum_{k \in K} \sum_{i \in I_{k}^{k}} b_{* i}^{k} \omega_{i}^{k}-\sum_{k \in K} \sum_{i \in I_{k}^{k}} b_{i}^{* k} t_{i}^{k} \longrightarrow \max , \\
u_{i}^{k}-\mu_{i j}^{k} u_{j}^{k}+\sum_{p=1}^{q} \lambda_{i j}^{k p} r_{p}-v_{i j} \leq c_{i j}^{k}, v_{i j} \geq 0, k \in K_{0}(i, j),(i, j) \in U_{0}, \\
u_{i}^{k}-\mu_{i j}^{k} u_{j}^{k}+\sum_{p=1}^{q} \lambda_{i j}^{k p} r_{p}-\omega_{i j}^{k} \leq c_{i j}^{k}, \omega_{i j}^{k} \geq 0, k \in K_{1}(i, j),(i, j) \in U, \\
u_{i}^{k}-\mu_{i j}^{k} u_{j}^{k}+\sum_{p=1}^{q} \lambda_{i j}^{k p} r_{p} \leq c_{i j}^{k}, k \in K(i, j) \backslash K_{1}(i, j),(i, j) \in U \backslash U_{0}, \\
-u_{i}^{k}+\sum_{p=1}^{q} \lambda_{i}^{k p} r_{p}+\omega_{i}^{k}-t_{i}^{k}=c_{i}^{k}, \omega_{i}^{k} \geq 0, t_{i}^{k} \geq 0, i \in I_{k}^{*}, k \in K,
\end{gathered}
$$


where the vector $\lambda=\left(u_{i}^{k}, k \in K, i \in I^{k} ; r_{p}, p=\overline{1, l} ; v_{i j} \geq 0,(i, j) \in U_{0} ; \omega_{i j}^{k} \geq 0,(i, j) \in U, k \in K_{1}(i, j) ; \omega_{i}^{k} \geq 0, t_{i}^{k} \geq\right.$ $\left.0, i \in I_{k}^{*}, k \in K\right)$ is a feasible solution of the dual problem (34) - (38).

Now we formulate the inverse optimization problem for the modeling of the parameters of the objective function for the primal problem (1) - (7). Let $x=\left(x_{i j}^{k},(i, j) \in U, k \in K(i, j) ; x_{i}^{k}, i \in I_{k}^{*}, k \in K\right)$ is a feasible solution of the problem (1) - (7). It is necessary to adjust the values of the components of the cost vector $c=\left(c_{i j}^{k},(i, j) \in U, k \in\right.$ $\left.K(i, j) ; c_{i}^{k}, i \in I_{k}^{*}, k \in K\right)$ so that the feasible solution $x$ becomes the optimal solution of the problem (1) - (7) for new values of the cost vector. Depending on its configured of values $x_{i j}^{k},(i, j) \in U, k \in K(i, j)$ and $x_{i}^{k}, i \in I_{k}^{*}, k \in K$ of the given feasible solution $x$ of the problem (1) - (7) we define the sets:

$$
\begin{gathered}
B_{1}=\left\{(i, j)^{k}, k \in K_{0}(i, j),(i, j) \in U_{0}: x_{i j}^{k}=0, \quad \sum_{k \in K_{0}(i, j)} x_{i j}^{k}=d_{i j}^{0}\right\}, \\
B_{2}=\left\{(i, j)^{k}, k \in K_{0}(i, j),(i, j) \in U_{0}: x_{i j}^{k}=0, \sum_{k \in K_{0}(i, j)} x_{i j}^{k}<d_{i j}^{0}\right\}, \\
B_{3}=\left\{(i, j)^{k}, k \in K_{0}(i, j),(i, j) \in U_{0}: x_{i j}^{k} \neq 0, \sum_{k \in K_{0}(i, j)} x_{i j}^{k}=d_{i j}^{0}\right\}, \\
B_{4}=\left\{(i, j)^{k}, k \in K_{0}(i, j),(i, j) \in U_{0}: x_{i j}^{k} \neq 0, \sum_{k \in K_{0}(i, j)} x_{i j}^{k} \neq d_{i j}^{0}\right\} ; \\
R_{1}=\left\{(i, j)^{k}, k \in K_{1}(i, j),(i, j) \in U: x_{i j}^{k}=0\right\}, \\
R_{2}=\left\{(i, j)^{k}, k \in K_{1}(i, j),(i, j) \in U: x_{i j}^{k}=d_{i j}^{k}\right\}, \\
R_{3}=\left\{(i, j)^{k}, k \in K_{1}(i, j),(i, j) \in U: 0<x_{i j}^{k}<d_{i j}^{k}\right\} ; \\
M_{1}=\left\{(i, j)^{k}, k \in K(i, j) \backslash K_{1}(i, j),(i, j) \in U \backslash U_{0}: x_{i j}^{k}=0\right\}, \\
M_{2}=\left\{(i, j)^{k}, k \in K(i, j) \backslash K_{1}(i, j),(i, j) \in U \backslash U_{0}: x_{i j}^{k}>0\right\} ; \\
M_{1}=\left\{i \in I_{k}^{*}, k \in K: x_{i}^{k}, k \in K: b_{* i}^{k}\right\},
\end{gathered}
$$

Applying the principle of inverse optimization we change the parameters of the objective function of the problem (1) - (7) in accordance with the following norm $l_{1}$ a sum of vectors:

$$
l_{1}=\sum_{(i, j) \in U} \sum_{k \in K(i, j)}\left|\alpha_{i j}^{k}-\beta_{i j}^{k}\right|+\sum_{k \in K} \sum_{i \in I_{k}^{t}}\left|\alpha_{i}^{k}-\beta_{i}^{k}\right|=\sum_{(i, j) \in U} \sum_{k \in K(i, j)}\left(\alpha_{i j}^{k}+\beta_{i j}^{k}\right)+\sum_{k \in K} \sum_{i \in I_{k}^{*}}\left(\alpha_{i}^{k}+\beta_{i}^{k}\right)
$$


where values increase $\alpha_{i j}^{k}$, decrease $\beta_{i j}^{k}$ of each parameter $c_{i j}^{k}$ and increase $\alpha_{i}^{k}$, decrease $\beta_{i}^{k}$ of each parameter $c_{i}^{k}$ of the objective function (1) can not be positive numbers simultaneously, where $\alpha_{i j}^{k} \geq 0, \beta_{i j}^{k} \geq 0,(i, j) \in U, k \in K(i, j)$, $\alpha_{i}^{k} \geq 0, \quad \beta_{i}^{k} \geq 0, \quad i \in I_{k}^{*}, \quad k \in K$.

Mathematical model of inverse optimization problem for modeling new parameters $\widetilde{c}=\left(c_{i j}^{k},(i, j) \in U, k \in\right.$ $\left.K(i, j) ; \widetilde{c}_{i}^{k}, i \in I_{k}^{*}, k \in K\right), \quad \widetilde{c}_{i j}^{k}=c_{i j}^{k}+\alpha_{i j}^{k}-\beta_{i j}^{k}, \quad \widetilde{c}_{i}^{k}=c_{i}^{k}+\alpha_{i}^{k}-\beta_{i}^{k}$ in accordance with the norm $l_{1}$ has the form:

$$
\begin{gathered}
\sum_{(i, j) \in U} \sum_{k \in K(i, j)}\left(\alpha_{i j}^{k}+\beta_{i j}^{k}\right)+\sum_{k \in K} \sum_{i \in I_{k}^{k}}\left(\alpha_{i}^{k}+\beta_{i}^{k}\right) \longrightarrow \min \\
u_{i}^{k}-\mu_{i j}^{k} u_{j}^{k}+\sum_{p=1}^{q} \lambda_{i j}^{k p} r_{p}-v_{i j} \leq c_{i j}^{k}+\alpha_{i j}^{k}-\beta_{i j}^{k}, v_{i j} \geq 0 \text { for the arcs }(i, j)^{k} \in B_{1} ; \\
u_{i}^{k}-\mu_{i j}^{k} u_{j}^{k}+\sum_{p=1}^{q} \lambda_{i j}^{p} r_{p} \leq c_{i j}^{k}+\alpha_{i j}^{k}-\beta_{i j}^{k} \text { for the arcs }(i, j)^{k} \in B_{2} ; \\
u_{i}^{k}-\mu_{i j}^{k} u_{j}^{k}+\sum_{p=1}^{q} \lambda_{i j}^{p} r_{p}-v_{i j}=c_{i j}^{k}+\alpha_{i j}^{k}-\beta_{i j}^{k}, v_{i j} \geq 0 \text { for the arcs }(i, j)^{k} \in B_{3} ; \\
u_{i}^{k}-\mu_{i j}^{k} u_{j}^{k}+\sum_{p=1}^{q} \lambda_{i j}^{p} r_{p}=c_{i j}^{k}+\alpha_{i j}^{k}-\beta_{i j}^{k} \text { for the arcs }(i, j)^{k} \in B_{4} ; \\
u_{i}^{k}-\mu_{i j}^{k} u_{j}^{k}+\sum_{p=1}^{q} \lambda_{i j}^{p} r_{p}-\omega_{i j}^{k} \leq c_{i j}^{k}+\alpha_{i j}^{k}-\beta_{i j}^{k} \omega_{i j}^{k} \geq 0 \text { for the arcs }(i, j)^{k} \in R_{1} ; \\
u_{i}^{k}-\mu_{i j}^{k} u_{j}^{k}+\sum_{p=1}^{q} \lambda_{i j}^{p} r_{p}-\omega_{i j}^{k}=c_{i j}^{k}+\alpha_{i j}^{k}-\beta_{i j}^{k}, \omega_{i j}^{k} \geq 0 \text { for the arcs }(i, j)^{k} \in R_{2} ; \\
u_{i}^{k}-\mu_{i j}^{k} u_{j}^{k}+\sum_{p=1}^{q} \lambda_{i j}^{p} r_{p}=c_{i j}^{k}+\alpha_{i j}^{k}-\beta_{i j}^{k} \text { for the arcs }(i, j)^{k} \in R_{3} ; \\
-u_{i}^{k}+\sum_{p=1}^{q} \lambda_{i}^{k p} r_{p}+\omega_{i}^{k}=c_{i}^{k}+\alpha_{i}^{k}-\beta_{i}^{k}, \omega_{i}^{k} \geq 0, \alpha_{i}^{k} \geq 0, \beta_{i}^{k} \geq 0 \text { for the } n o d e s i \in M_{1} ; \\
-u_{i}^{k}+\sum_{p=1}^{q} \lambda_{i}^{k p} r_{p}-t_{i}^{k}=c_{i}^{k}+\alpha_{i}^{k}-\beta_{i}^{k}, t_{i}^{k} \geq 0, \alpha_{i}^{k} \geq 0, \beta_{i}^{k} \geq 0 \text { for the } n o d e s i \in M_{2} ; \\
u_{i}+\sum_{p=1}^{q} \lambda_{i}^{k p} r_{p}=c_{i}^{k}+\alpha_{i}^{k}-\beta_{i}^{k}, \alpha_{i}^{k} \geq 0, \beta_{i}^{k} \geq 0 \text { for the } \text { nodes } i \in M_{3} . \\
u_{i}^{k}-\mu_{i j}^{k} u_{j}^{k}+\sum_{p=1}^{k} \lambda_{i j}^{p} r_{p}=c_{i j}^{k}+\alpha_{i j}^{k}-\beta_{i j}^{k}, \text { for the arcs }(i, j)^{k} \in L_{2} ; \\
\lambda_{i j} r_{p} \leq c_{i j}^{k}+\alpha_{i j}^{k}-\beta_{i j}^{k}, \text { for the arcs }(i, j)^{k} \in L_{1} ;
\end{gathered}
$$

The unknowns of the inverse optimization problem (39) - (43) are the values of the increase and the decrease of each parameter of the objective function (1). Decrease and increase of each parameter can not be positive numbers 
simultaneously. The parameters of the objective function (1) adjusted as little as possible in accordance with the following norm $l_{1}$ a sum of vectors so that the feasible solution $x=\left(x_{i j}^{k},(i, j) \in U, k \in K(i, j) ; x_{i}^{k}, i \in I_{k}^{*}, k \in K\right)$ becomes the optimal solution of the problem (1) - (7) for the new parameters $\widetilde{c}=\left(\widetilde{c}_{i j}^{k},(i, j) \in U, k \in K(i, j) ; \widetilde{c}_{i}^{k}, i \in\right.$ $\left.I_{k}^{*}, k \in K\right)$.

\section{REFERENCES}

[1] L. A. Pilipchuk. Linear Nonhomogeneous Problems in Network Flow Programming. Minsk, BSU, 2009, 222 pp. (in Russian)

[2] L. A. Pilipchuk. Sparse Underdetermined Systems of Linear Algebraic Equations. Minsk, BSU, 2012, 260 pp. (in Russian).

[3] L. A. Pilipchuk. Sparse Linear Systems and Their Applications. Minsk, BSU, 2013, 235 pp.

[4] Burton D., Toint Ph. L. Mathematical Programming. Vol. 53, No. 2. (1992), pp. 45-61.

[5] Ahuja R. K., Orlin J. B. Operation Research.Vol. 49, (2001), pp. 771-783.

[6] Jain S., Arya N. Mathematical Programming. Vol. 5, No. 4. (2013), pp. 24-27. 